\title{
Thanks to JMCP Peer Reviewers, 2016
}

The Journal of Managed Care \& Specialty Pharmacy would like to thank the 394 reviewers who have evaluated manuscripts in 2016 and contributed to the high quality of articles in JMCP. Without this excellent level of reviewer involvement, $J M C P$ would not be one of the most respected journals in health care services, connecting authors and innovators in order to accelerate advances in managed care pharmacy.

\section{—Laura E. Happe, PharmD, MPH Editor-in-Chief}

Ivo Abraham, RN, PhD

Susan Abughosh, PhD

Stacey J. Ackerman, MSE, PhD

Ashish Advani, PharmD

Ana M. Advinha, PharmD, PhD (candidate)

Parul Agarwal, PhD, MPH

Patricia Melo Aguiar, PhD

Vishal Ahuja, PhD

James Daniel Allen, PharmD

Aylin E. Altan, PhD

Miriam Alvarez-Payero, MS

JaeJin An, BPharm, PhD

Rajender Aparasu, $\mathrm{PhD}$

Sheta Ara, PharmD

Christine Leigh Bagley, PharmD

Simone Baldovino, MD

Mitchell J. Barnett, PharmD, MS

Christopher Bell, MS

Sandra Benavides, PharmD

Kristen K. Bender, RPh, PharmD

John P. Bentley, MBA, PhD

Todd Berner, MD

Gary Besinque, PharmD, FCSHP

Brookie M. Best, PharmD, MAS

Maribeth M. Bettarelli, PharmD, RPh

Nella Bieszk, PharmD

Joseph Biskupiak, PhD, MBA

Mary Ellen Bonk, PharmD

Jonathan R. Bouchard, MS, RPh

Joshua David Brown, PharmD, PhD

Mark N. Brueckl, BSPharm, MBA

Crystal J. Bryner, RPh, MBA

Ami Buikema, MPH

Elizabeth Marie Sutton Burke, PharmD

Sharon Burks, PharmD

Jonathan D. Campbell, PhD

H. Eric Cannon, PharmD

Karen Carroll, PharmD

Julian Casciano, BS
Jo-Ann L. Caudill, RPh

Laurie Michelle Cavendish, PharmD

Swetha Challagulla, MS

Carol Chan, PharmD

James Chan, PharmD, PhD

Elizabeth Chang, PharmD, PhD

Hsien-Yen Chang, PhD

Kevin Chang, PharmD

Kristen Dee Charlson, PharmD

Satabdi Chatterjee, MS, PhD

Shraddha Chaugule, PhD

Anita Chawla, PhD

Judy W. Cheng, PharmD

Sumit Chhabra, BPharm, MS, RPh

Donna Chiefari, BScPharm, PharmD

Chanadda Chinthammit, BSPharm, MS

Amy Chuang, PharmD

Shelley Chun, PharmD

Robert M. Cisneros, PhD

Zoe Clancy, PharmD

Karen M. Clements, ScD

John Coleman, PharmD

Candyce Collins, PharmD

Sandy Connell, RPh

Todd Cooperman, PharmD, MBA

Kenny Correia, PharmD

Joseph Edward Couto, PharmD

Jordan R. Covvey, PharmD, PhD, BCPS

William Crown, PhD

Tim Crum, PharmD

Eric J. Culley, PharmD, MBA

Dan Dalton, BS, MSPharm

Desola Davis, PharmD

Margaret H. Davis, MHS

Mary Lynn Davis-Ajami, PhD, MBA, MS, NP-C

Channel De Leon, PharmD, CGP

Pooja R. Desai, MS

Christopher Diehl, PharmD, MBA

Thomas J. Dilworth, PharmD
Qian Ding, PhD

Alexander James Dong, PharmD

Mary Dorholt, PharmD

Aaron Douglas Drovandi, MPharmPH

Emily Durden, $\mathrm{PhD}$

Michael Durkin, MS

Joseph Ted Dye, PhD, RPh

Keith Eaton, MD, PhD

Lisa A. Edwards, PharmD, RPh

Edward Eisenberg, MD

Maguy EL Hajj, PharmD, BCPS

Jeffrey J. Ellis, PharmD, MS

Sara Carruth Erickson, PharmD

Albert John Farias, PhD, MPH

Bithia Fikru, PharmD, MPA

Bridget M. Flavin, PharmD

Renee R. Fleming, RPh, MBA

Eileen Fonseca, MS

Sara Forrester, PharmD, MS

Sharon Glave Frazee, PhD, MPH

Raulo S. Frear, PharmD

Dustin D. French, PhD

Maria M. Garcia, MD, MPH, FACP

Justin Gatwood, PHD, MPH

William M. Gentry, PharmD

David A. Gettman, PhD, MBA, BSPharm

Teresa Gibson, PhD

Monika Gil, PharmD

Jasjit Gill, BSPharm, PharmD

Kimberly Gittings, PharmD (candidate)

Peter Glassman, MBBS, MSc

Patrick P. Gleason, PharmD, BCPS

Chester B. Good, MD, MPH

J. Kelly Goode, PharmD

Michael Grabner, PhD

Jennifer S. Graff, PharmD

Bonnie C. Greenwood, PharmD, BCPS

Joseph A. Gricar, MS

Sara Griesbach, PharmD, BCPS, BCACP 


\section{Thanks to JMCP Peer Reviewers, 2016 (continued)}

Paul 0. Gubbins, PharmD

Rachel Halpern, PhD, MPH

Mohamed G. Hashem, PharmD

Amany K. Hassan, PhD

Noelle K. Hasson, PharmD

Lindy Haynes, PharmD

Henry Henk, PhD

Ji Haeng Heo, MPharm

Zsolt Hepp, PharmD

Erin R. Himes, PharmD

Ana L. Hincapie, PhD, MS

Jennifer A. Hinnenthal, MPH

Mei Jen Ho, PharmD, MSPH

Janice Hoffman, PharmD

Jane Hoh, PharmD

Lisa Holle, PharmD, BCOP, FHOPA

Sari Hopson, PhD

Kristin Horning, PharmD

Rita L. Hui, PharmD, MS

Anna Hung, PharmD

Der-Wei Hwu, MD

Eric J. Ip, PharmD

Tara Kukuschkin Jellison, PharmD, MBA

Ruixuan Jiang, BS

Yawen Jiang, $\mathrm{PhD}$

Tianze Jiao, PhD

Shu Jing, PharmD

Amanda Johnson, PharmD, BCACP

Sejal A. Jonas, PharmD

Tina Joseph, PharmD, BCACP

Deborah Taira Juarez, ScD

Jeah (Kyoungrae) Jung, PhD

Stephen Jung, PharmD

Abby A. Kahaleh, BPharm, MS, PhD, MPH

Natan R. Kahan, RPh, MHA, PhD

Pravin Shivaji Kamble, BPharm, MTech,

RPh, MS, PhD

Anuraag Kansal, PhD

Sean Kearns, PhD

Shellie L. Keast, PharmD, PhD

James Kenney, BS, MBA

Alexandra Khachatryan, MPH

Robert Klein, MS

Donald G Klepser, PhD, MBA

Casey Ryan Koch, PharmD

Mark J. Kohn, PharmD, BCPS

Alexis Krumme, MS
Peter N. Kwok, PharmD

Maureen Lage, $\mathrm{PhD}$

Daniel C. Lane, PharmD, PhD

James Richard Lang, BSPharm, PharmD, MBA

Kourtney Laplant, PharmD, BCOP

Julie C. Lauffenburger, PharmD, PhD

Ken A. Lawson, PhD

Phuc Le, PhD, MPH

Catherine Lee, PharmD

Jeff Lee, PharmD, FCCP

Peter J. Lee, PharmD, MBA

Thomas S. Lee, PharmD

YoonJung Lee, PharmD

Kimberly Lenz, PharmD

Christine Leong, PharmD

Edward Li, PharmD, BCOP

Haitao Li, PharmD, MS

Joshua N. Liberman, PhD

Alexandra Tungol Lin, PharmD

Jay Lin, PhD, MBA

Vincent Lin, PharmD, MS

You-Li Ling, MS

Frank Xiaoqing Liu, PhD

Yifei Liu, PhD

Amanda Winters Livezey, PharmD

Terrie Livingston, PharmD

Carroline Priya Lobo, MS, PhD (candidate)

F. Ellen Loh, BSPharm, PhD, MBA

Charron Long, PharmD

Anthony Louder, RPh, PhD

Gregory A. Low, BPharm, PhD

Alan Lyles, MPH, ScD, BSPharm, BA

Elizabeth A. MacLean, PharmD

Tatiana Makhinova, $\mathrm{PhD}$

Daniel C. Malone, PhD

Amanda Mann, PharmD

Hend Mansoor, PharmD

Douglas Mapel, MD

Bradley C. Martin, PharmD, PhD

Karen E. Martin, PharmD, MBA, CGP

Aman Deep Matharoo, PharmD

Anthony J. May, PharmD, MBA

Carrie McAdam-Marx, PhD, RPh

Lisa McCarthy, PharmD, MSc

Kevin McCloy, PharmD

Cara McDermott, PharmD, PhD

Leann McDowell, PharmD, MS
Scott Thomas McDowell, PharmD, BCPS, CDE

Colleen A. McHorney, PhD

Milena M. McLaughlin, PharmD, MSc

Trent McLaughlin, PhD

Darshan Mehta, MS

Hemalkumar B. Mehta, MS, PhD

Jyothi Menon, MSPharm

Andrew Merker, PharmD

Daryl E. Miller, PharmD

Lesley-Ann Miller, MS, PhD

Rick Miller, RPh, MBA, CSP

Beatriz Manzor Mitrzyk, PharmD, BCPS

Pilar Modamio, PhD

Penny Mohr, MA

Chad Moretz, ScD

Daniel Mulkerin, MD

Kiraat Divyang Munshi, PhD

Radhika Nair, PhD

Uche Anadu Ndefo, PharmD

Brand Newland, PharmD, MBA

Huong Nguyen, PharmD, MPH (candidate)

Lynda Nguyen, PharmD

Weiyi $\mathrm{Ni}, \mathrm{PhD}$

Candace M. Nichols, PharmD, BCPS

Stephanie Nichols, PharmD

Daryn K. Norwood, PharmD

James Notaro, RPh, PhD

Kyle D. Null, PharmD, PhD

Heather Nyman, PharmD

Terrence 0'Shea, BSPharm, PharmD

Kevin Odell, PharmD

Gary M. Oderda, PharmD, MPH

Richard L. Ogletree, Jr., PharmD

Robert L. Ohsfeldt, PhD

Abiola Oladapo, $\mathrm{PhD}$

Alexander M. Orr, PharmD

Gary Owens, MD

Nancy M. Pace, BS, PharmD, MBA

Janki M. Panchal, PhD

Alan Pannier, PharmD, MBA

Haesuk Park, PhD

Jiehye Sarah Park, PharmD

Si Yeon Park, BSPharm

Taehwan Park, PhD

Yujin Park, PharmD

Margaret K. Pasquale, PhD

Aditi Patel, PharmD 


\section{Thanks to JMCP Peer Reviewers, 2016 (continued)}

Nick C. Patel, PharmD, PhD

Sonal Patel, PharmD

Michael Pazirandeh, PharmD

Patricia J. Peeples, PhD, MS, RPh

Peter M. Penna, PharmD

Fred W. Peyerl, PhD

An T. Pham, PharmD, MBA, FAHA

Shibani Pokras, MPH

Thomas C. Pomfret, PharmD, MPH, BCPS

Vickie Pon, PharmD

Mindy Prasad, PharmD

Sarah Priddy, PhD

Lauren Pusateri, PharmD

Yanru Qiao, MS

Nabeel Qureshi, PharmD, MPH

Marsha A. Raebel, PharmD

Dennis William Raisch, PhD

Jigar Ramesh Rajpura, PhD

Kate L. Ramsay, PharmD

Jean Rao, PharmD

Nazia Rashid, PharmD, MS

Rafia S. Rasu, PhD

Neda Ratanawongsa, MD, MPH

Prabashni Reddy, PharmD

Claiborne (Gene) E. Reeder, RPh, PhD

Debi Reissman, PharmD

Jinma Ren, PhD

Andrew Renda, MD, MPH

Shannon L. Reynolds, MSPH

Robyn Richard, PharmD

Nathaniel Marc Rickles, PharmD, PhD

Catherine S. Riggs, PharmD

Scott M. Robertson, RPh, PharmD

Carly Rodriguez, PharmD

Rosa Rodriguez-Monguio, $\mathrm{PhD}$

M. Christopher Roebuck, MBA, PhD

Brent Rollins, PhD

Deanna M. Rowe, PharmD

Elan Rubinstein, PharmD, MPH

Jim Ruble, PharmD, JD

Lewis Ruff, BSc, MSc

Michael T. Rupp, PhD, BSPharm, FAPhA

Kira L. Ryskina, MD

Naomi C. Sacks, PhD

Qayyim Said, PhD

Prashant Sakharkar, PharmD, MPH

Mahsa Salsabili, PharmD
Jayashri Sankaranarayanan, MPharm, PhD

Evelyn Sarnes, PharmD, MPH

Brian Christopher Sauer, PhD

Vishal H. Saundankar, MS, BPharm

Timothy E. Sawyers, BSPharm, MBA

Lauren M. Scarpati, PhD

Michael J. Schmidt, PharmD, BS, PS

Jordana Kate Schmier, MA

Jordan Schultz, PharmD

Jordan Sedlacek, PharmD

Jodi B. Segal, MD, MPH

Todd Sega, PharmD

Jason Shafrin, PhD

Sanket Shah, MBBS

Xian Shen, PhD

Christina H. Sherrill, PharmD

Katarzyna Shields, PharmD, MBA

Kathleen Shoemaker, PharmD, MBA

Caitlin Shorette, PharmD

Bruce Sill, PharmD, MS

Jonathan Silver, MD, MS, MBA

Komal Singh, BPharm, MS, PhD

Jane Skov, PhD

Mary Buatti Small, MS

Jennifer G. Smith, PharmD

Leah S. Smith, PharmD, BCPS, CGP

Scott A. Soefje, PharmD, MBA

Todd D. Sorensen, PharmD

Marion Joy Spark, PhD

Michele M. Spence, PhD

Christina A. Spivey, PhD

Joshua J. Spooner, PharmD, MS

Richard H. Stanford, PharmD, MS

Marilyn R. Stebbins, PharmD

Craig Stephen Stern, RPh, PharmD, MBA

Debbie J. Stern, RPh

Samuel Stolpe, PharmD

Wenqing Su, MS

Brandon T. Suehs, PharmD, PhD

Kent Summers, PhD

Jason P. Swindle, PhD, MPH

Patty Taddei-Allen, PharmD

Ashley Thomas, PharmD

Tara M. Thomas, RPh

Daniel Mark Tomaszewski, PharmD, PhD

Ivy Q. Tonnu-Mihara, PharmD, MS

Jennifer Tran, PharmD
Josephine (Nhu) Tran, PharmD, MS

Anne N. Tran-Pugh, PharmD

Allison Trawinski, PharmD, MBA

Kimberly Tsai, PharmD

Luke S. Tso, PharmD

Liang-Ruey Tu, BS, MS, MBA

Ozgur Tunceli, PhD

Robin Turpin, PhD

Connie A. Valdez, PharmD, MSEd, BCPS

Ami Vyas, PhD, MS, MBA

Rolin L. Wade, RPh, MS

Scot Walker, PharmD, MS, BCPS, BCACP

Takova D. Wallace, PharmD

Amanda Waltemath, PharmD, MPH

Curtis Wander, PharmD

Weijia Wang, MS

Albert I. Wertheimer, BS, MBA, PhD

Laurie Wesolowicz, PharmD

Donna S. West-Strum, PhD

Ria Westergaard, PharmD

Kimberly Westrich, MA

Salisa C. Westrick, PhD

Annesha White, PharmD, MS, PhD

Marnie Wickizer, PharmD

Courtenay Wilson, PharmD

Matthew J. Witry, PharmD, PhD

Jenifer Wogen, MS

Crystal A. Wright, PharmD

Joanne Wu, MD, MS

Jun $\mathrm{Wu}, \mathrm{PhD}$

Tzu-Hua Wu, PhD

Zhimin Xiao, MD, MS, MPH

Jing Xie, MD, MPH, ScD

Ting $\mathrm{Xu}, \mathrm{MD}, \mathrm{PhD}$

Yihua $\mathrm{Xu}, \mathrm{PhD}$

Sendra Yang, PharmD

Winnie M. Yang, PharmD

Xiaoqin Yang, $\mathrm{PhD}$

Caitlin Yeseta, PharmD

David C. Young, PharmD

Wisam Younis, PharmD

Jing Yuan, BPharm

Kathy Zaiken, PharmD 